\title{
Prototype of a Desk-top WiTricity System for Powering House-hold Utility Equipments
}

\author{
Hema Ramachandran, Bindu G.R \\ Department of Electrical Engineering, College of Engineering, Kerala, 695014, India \\ E-mail:hemaachuth@gmail.com
}

\begin{abstract}
Magnetic resonant coupling systems have been in vogue for some years after it was demonstrated for mid-range power transfer in 2007. This paper proposes a prototype desk-top Witricity system for powering house-hold utility equipments. The system uses the phenomena of near-field resonance coupling achieved at $400 \mathrm{kHz}$ using a transmission coil wound around rim of a table and a receiver coil wound around the base of a white-LED lit table lamp. The parasitic capacitance of coils are calculated and augmented with external capacitance to achieve resonance. Distance does not come into play in this system while the system is of great practical use for household utility equipments such as table lamps mobile phones etc.
\end{abstract}

Keywords - Witricity; wireless charging; resonance coupling; near field coupling

\section{INTRODUCTION}

Wireless electricity (Witricity) uses the principle of magnetic resonance coupling to effect power transfer [1]. It uses the near field of high frequency magnetic coils in the range below $\frac{\lambda}{2 \pi}$, where lambda is the wavelength of the power supply. This field is a non-radiative evanescent field in contrast to a far field which is radiative. Wireless power transmission uses transmitter and receiver coils which have varying coil geometries [2]. The frequency is electromagnetically compatible in the radio frequency range. The coils transmit energy continuously at the resonant frequency while interacting weakly with other objects. The energy transfer diminishes as square of distance and this is found to be the limiting factor in real-life applications. The losses due to iron core are absent but inefficient linkage of magnetic field drastically reduces efficiency of the system. Inspite of this efficiency limitation, our attempt here is to show that the effect can be of practical use. The implementation of the prototype of a Witricity powered desk-top Witricity system for powering household utility devices like table lamps, and frequently charged devices like cell phones and laptops is described in this paper. Our innovation is limited to implementing the Witricity effect in a pragmatic way.

\section{CONCEPTUAL FRAMEWORK FOR MODELLING}

In our prototype, each turn of wire in the transmitter and receiver coils acts as a capacitor plate. The totality of the effects of each turn acts as distributed capacitance or self capacitance or parasitic capacitance. This is due to electric field between the turns which are at different potentials. At high frequencies in which Witricity operates, this capacitance will significantly contribute to the resonant mode of the coils. In addition, capacitance between the transmitter and receiver coils (inter-coil capacitance) also affects the behaviour of the resonant circuit. Fig. 1 shows the equivalent circuit of the system [7].

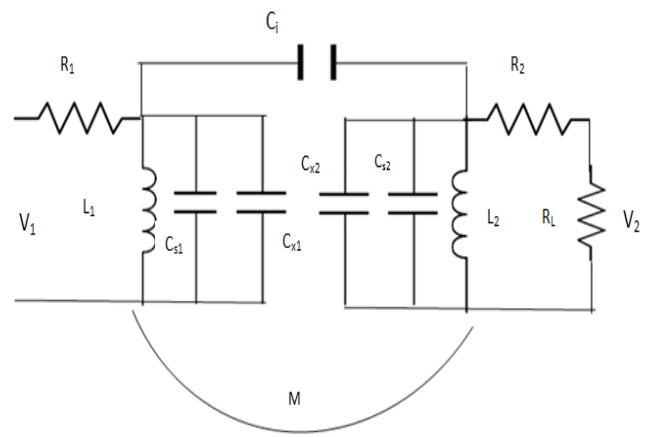

Fig.1 Equivalent circuit of the Witricity system:

$\mathrm{V}_{1}$-Applied voltage

$\mathrm{V}_{2}$-Voltage across the load

$\mathrm{R}_{1}, \mathrm{R}_{2}$ - Resistance of the inductive coils

$\mathrm{L}_{1}, \mathrm{~L}_{2}-$ Self inductances of the primary and secondary coils.

M- Mutual inductance between the primary and secondary coils.

$\mathrm{C}_{\mathrm{s} 1}, \mathrm{Cs}_{2}-$ Distributed capacitance across the primary and secondary coils.

$\mathrm{C}_{\mathrm{x} 1}, \mathrm{C}_{\mathrm{x} 2}$ - Additional capacitance to effect resonant tuning

$\mathrm{C}_{\mathrm{i}}$-interwinding capacitance

$\mathrm{R}_{\mathrm{L}}$ - load resistance 
In the lumped component model [3], the inductor has a self capacitance $\mathrm{C}_{\mathrm{s}}$ the parasitic capacitance of the measuring setup $C_{m}$ and a variable experimental parameter $C_{x}$. The total capacitance

$$
\mathrm{C}=\mathrm{C}_{\mathrm{s}}+\mathrm{C}_{\mathrm{m}}+\mathrm{C}_{\mathrm{x}}
$$

The resonant frequency of oscillation is given by

$$
f=1 /(2 \pi \sqrt{L C)}
$$

The first theoretical work for calculating parasitic capacitance was done on soft ferrites by E.C. Snelling [4] who recommends the use of Medhurst formula. Medhurst, in his experimental on solenoid coils with non-magnetic cores developed a mathematical formula for self capacitance on a number of test coils wound on polystyrene rods. His optimisation formula is

$$
\mathrm{C}_{\mathrm{L}}=\left(4 \varepsilon_{0} / \pi\right) l\left(1+0.842(\mathrm{D} / \mathrm{l})+2.329(\mathrm{D} / \mathrm{l})^{3 / 2}\right)
$$

where 1 is the solenoid length in metres and D is the average diameter of the solenoid in metres [5] This formula has been corrected by David W. Knight by the inclusion of $1 / \cos ^{2} \psi$ where $\varphi$ is the pitch angle. The self capacitance of a helical coil takes the form

$$
\mathrm{C}_{\mathrm{L}}=\left(4 \varepsilon_{0} / \pi\right) l\left(1+0.842(\mathrm{D} / \mathrm{l})+2.329(\mathrm{D} / 1)^{3 / 2)} \cos ^{2} \psi\right.
$$

The pitch angle [3] for a helical coil is related to the length of the wire 1 , effective radius $r$ of the solenoid and the number of turns $\mathrm{N}$ by the formula

$$
\cos \varphi=2 \pi \mathrm{rN} / l
$$

The parasitic capacitance together with the additional capacitance will resonate with the transmitter inductive coil throwing it to resonance.

If capacitance is taken as the means of tuning, the coil winding parameters can be chosen freely, taking practical issues into consideration. The coil inductance L of an aircored helical coil can be computed by

$$
\mathrm{L}=\mathrm{R}^{2} \mathrm{~N}^{2} /(9 \mathrm{R}+10 \mathrm{~h})
$$

where $\mathrm{R}$ is the radius of the coil in inches, $\mathrm{N}$ is the number of turns and $h$ is the height of the wire in inches [6]. In our prototype, as the frequency is chosen $400 \mathrm{kHz}$ for resonance of the transmitter the capacitance of the receiving coil is adjusted with an external additional capacitance to achieve resonance. This frequency is not claimed to be optimal. Experiments are ongoing to find the optimal frequency.

\section{Q FACTOR AND EFFECIENCY}

The resonant behaviour of coils acts in a manner as to selectively respond to signal of a particular frequency and discriminating signals of all other frequencies. Quality factor is a measure of this selectivity. Quality factor (Q) at resonance can be expressed as

$$
\mathrm{Q}=\mathrm{X}_{\mathrm{L}} / \mathrm{R}=-\mathrm{X}_{\mathrm{C}} / \mathrm{R}=(1 / \mathrm{R}) \sqrt{\mathrm{L}} / \mathrm{C}
$$

where $R$ is the total resistance, $X_{L}$ and $X_{c}$ are the inductive reactance and capacitive reactance of the coils. In Witricity systems, the quality factor of the transmitter and receiver coils are very high so that efficiency is increased. The efficiency of power transfer is

$$
\eta=\frac{r_{t}^{2} r_{r}^{2} Q_{t Q R K t K r}}{16 d^{6}}
$$

where $r_{t}$ and $r_{r}$ are the radius of the transmitter and receiver coils, $\mathrm{K}_{\mathrm{t}}$ and $\mathrm{K}_{\mathrm{r}}$ are the coil geometry terms, $\mathrm{Q}_{\mathrm{t}}$ and $\mathrm{Q}_{\mathrm{r}}$ are the quality factors of the transmitter and receiver coils and $\mathrm{d}$ is the transfer distance[7]. It has been found that capacitively-loaded conducting wire loops are found to increase the quality factor. The resonant tuning requires calculation of the parasitic capacitance for further correction by addition of extra capacitors. These capacitors can be included in the transmitter and receiver coil circuitry to increase coupling. The distributed capacitance caused by the turns layered on top of each other acts in parallel with the inductance of coils on the transmitter and receiver coils.

\section{THE DESK-TOP WITRICITY SYSTEM PROTOTYPE}

For implementing a prototype, a traditional Indian cane table of diameter 0.63 meters is chosen. We wound a transmitter coil of 4 turns around the rim of the table. A receiver coil of 35 turns is wound around the base of a white LED lit table lamp of base diameter 0.13 meters. The inductance and the parasitic capacitance of the transmitter coil are calculated and the resonant frequency is noted as nearly $400 \mathrm{kHz}$. The system is then made to resonate by adjusting the capacitance of the receiving coil. A standard Colpitts oscillator is used for exciting the transmitter coil. To achieve the required power level a Class $\mathrm{AB}$ amplifier is used. Fig.3. shows the system in operation.

The variation of inductance and parasitic capacitance of a circular coil with number of turns and also diameter of the coil, as per equations given above are shown graphically. This serves as a design guide for coils in the proposed system. This is done in MATLAB environment.

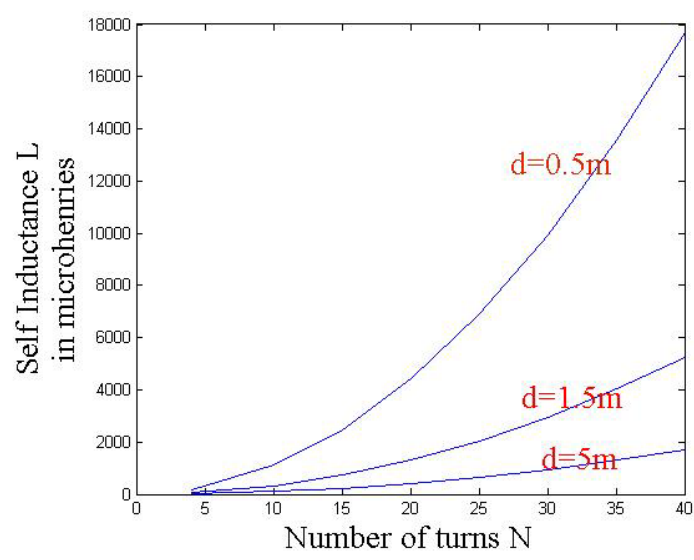

Fig. 2(a) Graph showing variation of inductance d of coils with the number of turns 


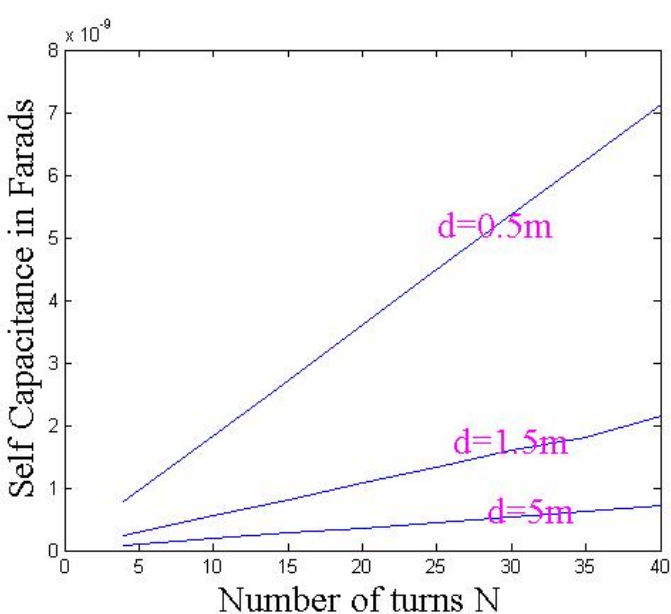

Fig. 2(b) Graph showing variation of parasitic capacitance of coils with the number of turns

There is no hysteresis or eddy current losses in Witricity systems as no metal core is used. This will increase the magnetising current, for the given flux density. In the nearfield, field strength decreases rapidly as the square of distance from the transmitter; this would be the major determinant of efficiency. In the particular design proposed, the distance does not play a major role as the receiver coil is on the table top and the transmitter coil is just beneath it. From this angle, the power transfer efficiency is very high. At high frequencies, inductive coils also encounter two types of losses on account of skin effect and proximity effect. It can create power loss in Witricity systems and also reduce Q factor. Skin effect is due to increased resistance of a wire at high frequency. Current at high frequencies travels along the body of the conductor without penetrating into the inside of the conductor. Thus the entire cross sectional area is not used for conducting current. This increases the resistance of wire due to the lesser diameter of the conductor. The depth at which the current penetrates

$$
\delta=\sqrt{(2 \rho} / \omega \mu)
$$

where $\rho$ is the resistivity of the conductor, $\omega$ is the angular frequency of current and $\mu$ is the absolute permeability of the conductor. Proximity effect occurs in conducting wires which are very close to each other. The magnetic field of the adjacent turns induces eddy currents in the coil, which causes concentration of current in a thin area near the adjacent wire. The cross sectional area of the conductor is reduced thereby increasing resistance.
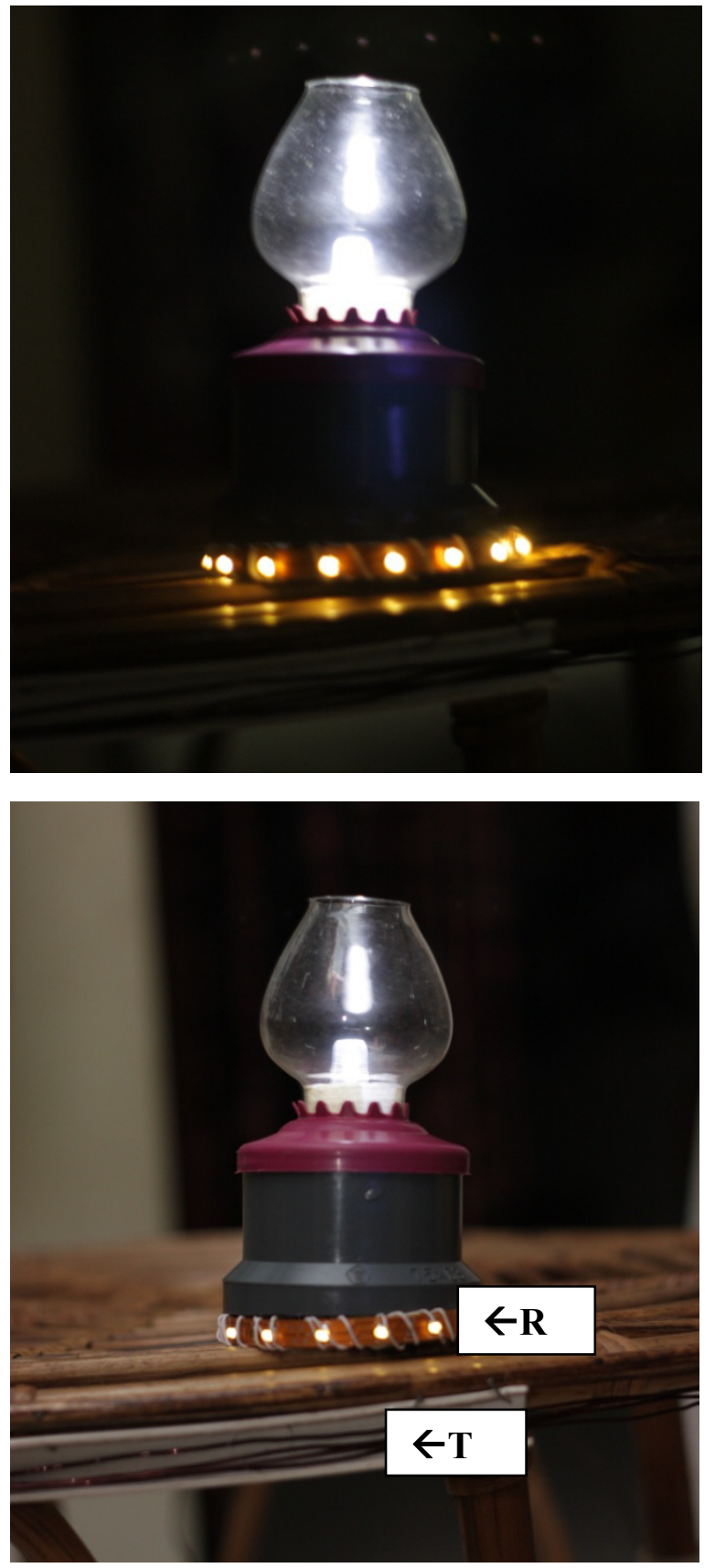

Fig.3. Desk-top Witricity system in operation photographed without camera flash (top) and photographed with camera flash (bottom). Transmitter coil wound on the rim of the cane table is marked $\mathrm{T}$ and the receiver coil is marked $\mathrm{R}$. The receiver coil powers the table lamp as well as the decorative LEDs at the base of the lamp.

\section{CONCLUSIONS AND FUTURE SCOPE}

The system described in this paper demonstrates the feasibility of using Witricity systems for utility house-hold purposes. The prototype described in this paper is not an optimal design, but a proof of concept and further experimentation is ongoing. Computation of resonance parameters in the equivalent circuit forms the core design approach. The fine tuning of the $\mathrm{Q}$ factor needs to be analysed further when the design is scaled up. As in any Witricity system, though efficiency is a limiting parameter, in this particular case the distance between transmitter and receiver coils is not a limiting parameter. Experimental 
studies on analysing the efficiency of the system and the relation to resonance frequencies and other parameters is ongoing. The successful performance of the system indicates the possibility of a scaling up.

\section{ACKNOWLEDGEMENT}

We acknowledge the valuable comments and inputs given by David W. Knight, Prakash Chandran and Sundara Vadanan. The first author acknowledges support from Speed IT Research Fellowship from IT Department of the Govt of Kerala. An Indian patent is pending for the innovation described in this paper.

\section{REFERENCES}

[1] A.Kurs, A.Karalis, R. Moffat, J.D. Joannopoulos, P. Fischer and M.Soljacic, "Wireless power transfer via strongly coupled magnetic resonances”, Science, Vol. 317, pp 83-86, Jul 2007.

[2] A Karalis, J.D. Joannopoulos and M. Soljacic, "Efficient wireless non-radiative mid-range energy transfer", Annals of Physics, Vol. 323, pp 34-48, Jan2008.

[3] David W Knight, A G Kandoian, W Sichak, "The self-resonance and self capacitance of solenoid coils", Electrical Communication 30(4) pp 294-299, 1953..

[4] E.C Snelling, "Soft Ferrites, Properties and Applications", $2^{\text {nd }}$ edition, Butterworth, 1988 pp 330-335.

[5] R.G. Medhurst, "High Frequency Resistance and Self-capacitance of single layer solenoids" Wireless Engineer 24(281):35-43, Feb 1947.

[6] Frederick W. Grover, “ Inductance Calculations”, Dover Publications, New York, 1952

[7] Niegel P. Cook et al, U. S patent 20090079268A1(2009). 